\title{
Intralobar Pulmonary Sequestration Showing Increased Serum CA19-9
}

Yong Hwan Ahn, M.D. ${ }^{1}$, Mi Jin Song, M.D. ${ }^{1}$, Sang Hyun Park, M.D. ${ }^{2}$

Departments of ${ }^{1}$ Internal Medicine and ${ }^{2}$ Radiology, Plus Internal Medicine Clinic, Suncheon, Korea

Carbohydrate antigen 19-9 (CA19-9) is a specific tumor marker of the biliary, pancreatic and gastrointestinal tracts. CA19-9 is occasionally elevated in serum in patiens with benign pulmonary diseases such as bronchiectasis, idiopathic interstitial pneumonia or collagen disease-associated pulmonary fibrosis. Intralobar pulmonary sequestration is an uncommon congenital lung anomaly. It is dissociated from the normal tracheobronchial tree and is supplied by an anomalous systemic artery. There have been some reports of elevation of CA19-9 in this lesion. We report a case of intralobar pulmonary sequestration with elevated serum CA19-9 in a 29-year-old man who was diagnosed with bronchiectasia of left lower lung field on general check up. He had no evidence of any malignant disease in pancreatobiliary or gastrointestinal tracts. Elevated serum CA19-9 level might be encountered with benign pulmonary disease such as pulmonary sequestration.

Key Words: Bronchopulmonary Sequestration; CA-19-9 Antigen

\section{Introduction}

Pulmonary sequestration is a rare congenital anomaly of lung, in which pulmonary parenchyma is sequestered from normal bronchial trees, of which blood is supplied by systemic arteries ${ }^{1}$. In general, although such conditions as pulmonary infection are commonly associated clinical features, nonspecific or sometimes no symptoms may be found at initial diagnosis, and occasionally even misdiagnosed as pulmonary abscess or tumor, which is truly confirmed later and corrected postoperatively ${ }^{2}$. Although serum carbohydrate antigen 19-9 (CA19-9) is a tumor marker for pancreatobiliary and gastrointestinal tract, it can also be elevated in idiopathic interstitial pneumonia, collagen vascular disease associated lung diseases, diffuse panbronchiolitis, or bronchiectasis as well as lung cancer ${ }^{3,4}$. Recently, we have experienced a case of intralobar pulmonary sequestration accom-

Address for correspondence: Yong Hwan Ahn, M.D. Department of Internal Medicine, Plus Internal Medicine Clinic, 981, Jorye-dong, Suncheon 540-967, Korea

Phone: 82-61-804-5114, Fax: 82-61-804-5113

E-mail: spikeahn@naver.com

Received: Nov. 1, 2011

Revised: Nov. 21, 2011

Accepted: Dec. 7, 2011 panying with elevated serum CA19-9 level from a patient suspected of bronchiectasis in routine medical examination, and report here with review.

\section{Case Report}

A 29-year-old man visited our clinic for evaluation of abnormal finding in chest computed tomography (CT). One month ago visiting clinic, he had general check up in a medical examination center in Seoul. He was suspected of pneumonia and bronchiectasis in chest CT with elevated serum CA19-9 level. He was a company staff, a non-smoker and had no family history of pulmonary tuberculosis or bronchiectasis. He had no previous history of frequent pulmonary infection in his early years. At 20, he was diagnosed as pneumonia and admitted for 1 week without any information or comment on other pulmonary diseases. In the blood test from work place health examination checked 3 years and 1 year ago, CA19-9 level was found to be elevated over $300 \mathrm{U} / \mathrm{mL}$, followed by esophagogastroduodenoscopy (EGD), colonoscopy, abdominal ultrasonography and CT with no specific abnormal finding found. He has been on observation since then. He appeared relatively 
healthy and neither sign of fever, chills, weight loss nor pulmonary or digestive symptom was observed at the time of visit. His blood pressure was 138/81 mm Hg, pulse rate $72 / \mathrm{min}$, respiratory rate $20 / \mathrm{min}$, and body temperature $36.4^{\circ} \mathrm{C}$. There were no pains or rebound tenderness, or any palpable mass in the abdominal examination. No other specific abnormality was found other than minimal decrease of breathing sound in the left lower lung field in auscultation. All the result for

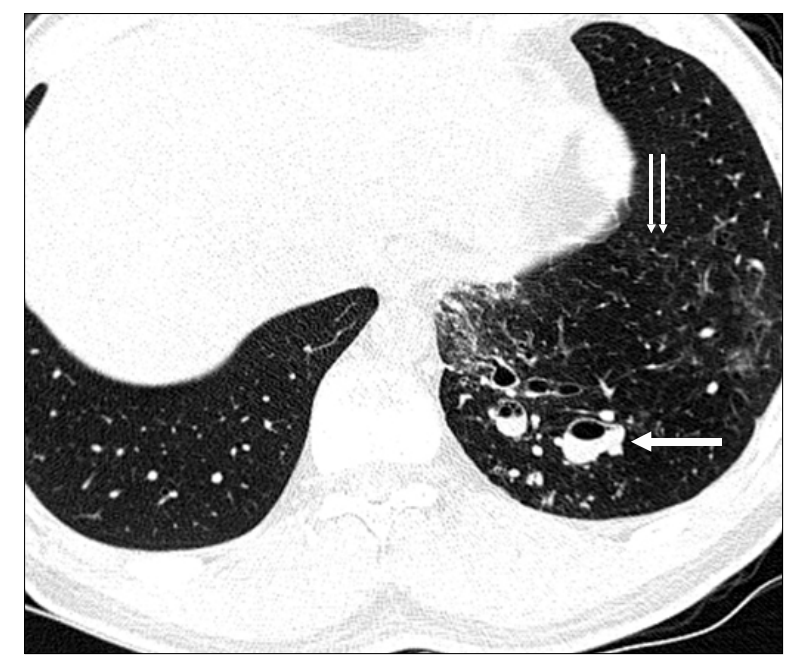

Figure 1. High-resolution computed tomography scan shows emphysematous change (double arrow) and bronchiectasis (arrow) in left lower lobe of lung.

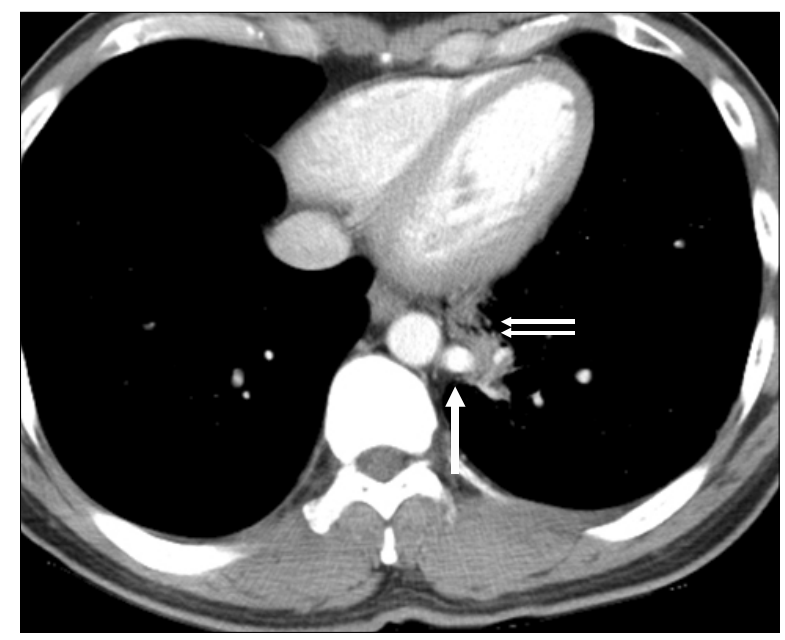

Figure 2. Contrast enhanced computed tomography demonstrates an anomalous artery (arrow) originating from the upper abdominal aorta and extending into the sequestered lung (double arrow). blood test checked in the examination center 1 month ago were normal except elevated CA19-9 level to 626 $\mathrm{U} / \mathrm{mL}$. Complete blood count result at the visit were normal (white blood cell 6,200/ $\mathrm{mm}^{3}$, hemoglobin 14.1 $\mathrm{g} / \mathrm{dL}$, and platelet $209,000 / \mathrm{mm}^{3}$ ). In the chemistry panel, aspartate aminotransferase $26 \mathrm{IU} / \mathrm{L}$, alanine aminotransferase $33 \mathrm{IU} / \mathrm{L}$, alkaline phosphatase $73 \mathrm{IU} / \mathrm{L}$, total protein $8.3 \mathrm{~g} / \mathrm{dL}$, albumin $4.8 \mathrm{~g} / \mathrm{dL}$, total bilirubin 0.6 $\mathrm{mg} / \mathrm{dL}$, amylase $44 \mathrm{U} / \mathrm{L}$, all of which were within reference range. Tumor markers tested at the same day with radioimmunoassay showed elevated CA19-9 level to 248.3 U/mL, but all the other results were within normal range (alpha fetoprotein $6.80 \mathrm{ng} / \mathrm{mL}$, carcinoembryonic antigen [CEA] $3.1 \mathrm{ng} / \mathrm{mL}, \mathrm{CA} 12524.4 \mathrm{U} / \mathrm{mL}$ ). No other specific abnormality was found other than chronic superficial gastritis in the examinations including EGD and colonoscopy. In the abdominal ultrasonography and CT, all finding in intrahepatic bile duct, common bile duct, and pancreas were normal. Chest high-resolution CT at the medical examination showed emphysematous change with bronchiectasis (Figure 1), which were similar to the chest CT findings checked again in our clinic.

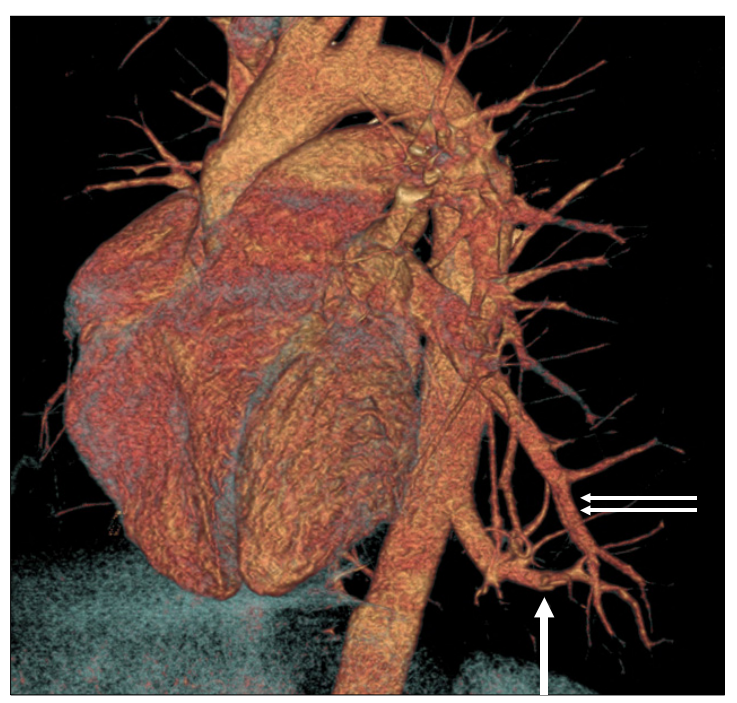

Figure 3. Volume rendering image demonstrates an artery (arrow) originating from the descending aorta and supplying the intralobar pulmonary sequestration. Also note a large draining vein (double arrow) originating from the sequestration and draining into the left inferior pulmonary vein. 
However, left to left shunt formed by artery supplying blood flow from descending aorta to left lower pulmonary lobe (Figure 2) and venous outflow to left inferior pulmonary vein was identified and diagnosed as intralobar pulmonary sequestration (Figure 3).

\section{Discussion}

Pulmonary sequestration is classified in two types, intralobar pulmonary sequestration in which normal pulmonary tissue and visceral pleura is shared, and extralobar pulmonary sequestration where pulmonary tissue is surrounded with the pleura of the lesion itself $f^{1,2}$. In intralobar pulmonary sequestration, some cases features with elevated CA19-9 level alone or together with the elevation of other tumor marker such as CEA or CA125 $5^{5-7}$, which is also reported in extralobar pulmonary sequestration $^{8}$. In the cases of elevated CA19-9 in patients with pulmonary sequestration, no abdominal symptoms were found and most cases were accidently identified by blood test for diagnosis of pulmonary sequestration or prior to surgery ${ }^{5-8}$. Although the exact cause for the condition is not yet, main mechanism is thought to be the effect of chronic inflammatory process of epithelial cells, which is based on the histologic findings of abundancy of mucus, identification of tumor marker response, and no signs for infection in the patients with remarkable elevation of CA19-9 after several years, who had normal level of CA19-9 at the time of initial identification of emphysematous lesion before ${ }^{5}$. According to other literature, tumor markers are reported to be synthesized in normal bronchial epithelial cells, and elevated in the blood by productive process of epithelial cells and accumulation in the pulmonary sequestration? ${ }^{\text {. Some other }}$ reports claims that the proliferation of bronchial epithelial cells is the cause for the elevation of tumor marker by chronic inflammatory process by infection of pathogenic organisms such as Aspergillus or Mycobacterium $^{10,11}$. However, in this case, although histologic confirmation of tumor markers through postoperative immunohistochemical staining or identification of infection of other organism in tissue was not available, tumor markers is thought to be elevated in the repetitive process of injury and recovery as chronic inflammatory process.

Over 50\% of the patients with pulmonary sequestration, cough, sputum, and frequent pulmonary infection are typically accompanied, but no symptoms could be found in many cases ${ }^{2}$. Although there still exists controversy whether to perform surgery or not in asymptomatic young patients as in this case, embolization of abnormal artery or active surgical intervention is required because serious condition could possibly happen such as hemoptysis or hemothorax, followed by rapid aggravation of disease course in the pulmonary sequestration $^{12,13}$. In the cases with elevation of tumor markers like CA19-9 without any other gastrointestinal diseases, identification of any hidden pulmonary diseases is required as fatal situation such as hemorrhage due to pulmonary sequestration could occur if unnoticed. And also, if ignorant of the association with this kind of benign pulmonary diseases, unnecessary examination for gastrointestinal diseases could be implemented by misidentification of gastrointestinal malignancy. Regarding pulmonary disease, such as bronchiectasis, one case was reported related to elevated CA19-9 in Korea ${ }^{14}$.

Decreased survival rate was reported in the group with higher CA19-9 level in idiopathic interstitial pneumonia and collagen vascular disease associated lung diseases, and the level of CA19-9 was expected to be related to disease activity in these condition ${ }^{4}$. However, further study on the association of survival rate with the tumor marker in pulmonary sequestration is required, as the disease was not included on this study. Furthermore, additional study on the association of the change of tumor marker level with diseases activity or prognosis in pulmonary sequestration is also required. Here we report a novel case of pulmonary sequestration showing increased CA19-9 with literature review, which has not yet been reported in Korea to our best knowledge.

\section{References}

1. Savic B, Birtel FJ, Tholen W, Funke HD, Knoche R. 
Lung sequestration: report of seven cases and review of 540 published cases. Thorax 1979;34:96-101.

2. Cho HM, Shin DH, Kim KD, Lee S, Chung KY. Clinicopathological correlation of intralobar pulmonary sequestration. Korean J Thorac Cardiovasc Surg 2003;36: $356-62$.

3. Gupta MK, Arciaga R, Bocci L, Tubbs R, Bukowski R, Deodhar SD. Measurement of a monoclonal-antibody-defined antigen (CA19-9) in the sera of patients with malignant and nonmalignant diseases: comparison with carcinoembryonic antigen. Cancer 1985;56:277-83.

4. Kodama T, Satoh H, Ishikawa H, Ohtsuka M. Serum levels of CA19-9 in patients with nonmalignant respiratory diseases. J Clin Lab Anal 2007;21:103-6.

5. Ambiru S, Nakamura S, Fukasawa M, Mishima O, Kuwahara T, Takeshi A. Intralobar pulmonary sequestration associated with marked elevation of serum carbohydrate antigen 19-9. Ann Thorac Surg 2009;88: 2010-1.

6. Yagyu H, Adachi H, Furukawa K, Nakamura H, Sudoh A, Oh-ishi S, et al. Intralobar pulmonary sequestration presenting increased serum CA19-9 and CA125. Intern Med 2002;41:875-8.

7. Uyama T, Monden Y, Harada K, Tsuzuki H, Hashioka $\mathrm{K}$, Nobuhara K, et al. A case of intralobar pulmonary sequestration with calcification and elevated serum values of carcinoembryonic antigen and carbohydrate antigen 19-9. J Thorac Imaging 1989;4:74-6.
8. Armbruster C, Kriwanek S, Feichtinger H, Armbruster C. Intra-abdominal sequestration of the lung and elevated serum levels of CA 19-9: a diagnostic pitfall. HPB (Oxford) 2004;6:45-8.

9. Matsuoka Y, Endo K, Kawamura Y, Yoshida T, Saga T, Watanabe Y, et al. Normal bronchial mucus contains high levels of cancer-associated antigens, CA125, CA19-9, and carcinoembryonic antigen. Cancer 1990; 65:506-10

10. Nagaoka $H$, Taniguchi $T$, Iesato $H$, Inoue $T$, Watanuki A, Yokomori T, et al. Pulmonary sequestration with the elevated serum value of CA19-9: a case report. J Jpn Pract Surg Soc 1996;57:571-4.

11. Ishiura $Y$, Fujimura $M$, Minami $S$, Ueda A, Iwata $M$, Watanabe $\mathrm{K}$, et al. Increased CA19-9 level in serum and brochoalveolar lavage fluid from a patient with pulmonary tuberculosis. Nihon Kyobu Shikkan Gakkai Zasshi 1996;34:477-81.

12. Park JM, Oh BS. Intralobar pulmonary sequestration with hemoptysis and hemothorax. Korean J Thorac Cardiovasc Surg 2007; 40:708-10.

13. Nakamura $\mathrm{H}$, Makihara $\mathrm{K}$, Taniguchi $\mathrm{Y}$, Ishiguro $\mathrm{K}$, Ohgi S. Thoracoscopic surgery for intralobar pulmonary sequestration. Ann Thorac Cardiovasc Surg 1999; 5:405-7.

14. Huh JH, Lee SM, Koo TH, Shin BC, Um SJ, Yang DK, et al. A case of bronchiectasis with high serum CA19-9. Tuberc Respir Dis 2008;65:383-6. 